\title{
Desktop Cartesian-Type Robot with Abilities of Compliant Motion and Stick-Slip Motion
}

\author{
Fusaomi Nagata, Shintaro Tani and Takanori Mizobuchi \\ Tokyo University of Science, Yamaguchi \\ Japan \\ Tetsuo Hase and Zenku Haga \\ RED Center, Meiho Co. Ltd., Fukuoka \\ Japan \\ Keigo Watanabe \\ Okayama University, Okayama \\ Japan
}

\section{Introduction}

In manufacturing process of small lens molds, 3D CAD/CAM systems and high precision NC (numerically controlled) machining centers are used generally, and these advanced systems have drastically rationalized the design and manufacturing process. For example, recently, an ultra precision multi-axis control machining system has been developed for spherical micro-lens array molds. As a result, the mold with a spherical micro lens array has been effectively shaped with high accuracy (Oba et al., 2008).

In case of an LED lens mold as shown in Fig. 1, however, the finishing process after the machining process has been hardly automated yet, because the LED lens mold has plural small concave areas to be finished, in which each diameter is $4 \mathrm{~mm}$.

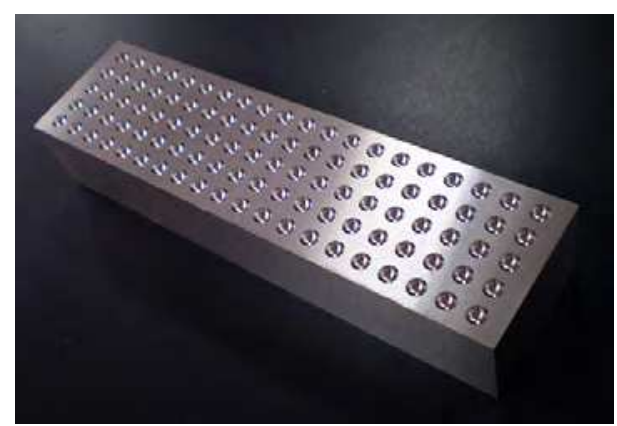

Fig. 1. Example of a target LED lens mold. Each diameter of concaved area is $4 \mathrm{~mm}$. 
That means the target mold is not axis-symmetric, so that it is difficult for conventional effective polishing systems (e.g., Kuriyagawa et al., 2003; Suzuki et al., 2006), which are good at dealing with axis-symmetric workpieces, to be applied to the LED lens mold well. Accordingly, such axis-asymmetric LED lens molds are finished by skilled workers in almost cases. The skilled workers usually finish a mold by using a small wood stick tool with diamond lapping paste, while qualitatively checking the concave surface through a microscope.

In this paper, a new desktop Cartesian-type robot, which has abilities of compliant motion and stick-slip motion, is first presented for finishing small metallic molds with curved surface. The Cartesian-type robot is also called the orthogonal-type robot. The robot consists of three single-axis robots with a high position resolution of $1 \mu \mathrm{m}$. A thin wood stick tool is attached to the tip of the z-axis. The tool tip has a small ball-end shape. The control system is composed of a force feedback loop, position feedback loop and position feedforward loop. The force feedback loop controls the polishing force consisting of tool's contact force and kinetic friction forces. The position feedback loop controls the position in spiral direction, i.e., z-direction. The position feedforward loop leads the tool tip along a desired trajectory called cutter location data (CL data). The CL data are generated from the main-processor of a CAM system. The proposed Cartesian-type robot has realized a compliant motion required for the surface following control along a spiral path.

In order to improve the finishing performance, a small stick-slip motion control strategy is further added to the control system. The small stick-slip motion is orthogonally generated to the direction of the tool moving direction. Generally, the stick-slip motion is an undesirable phenomenon and should be eliminated in precision machineries (Bilkay \& Anlagan, 2004; Mei et al., 2004). However, the proposed Cartesian-type robot employs a small stick-slip motion to improve the finishing quality. The effectiveness of the robot was examined through an actual finishing test of an LED lens mold with a diameter of $4 \mathrm{~mm}$. It was observed that the undesirable small cusps could be removed uniformly. Further, it was confirmed from the result that the proposed Cartesian-type robot with the abilities of compliant motion and stick-slip motion has a superior performance to achieve a higher quality surface like a mirror finishing.

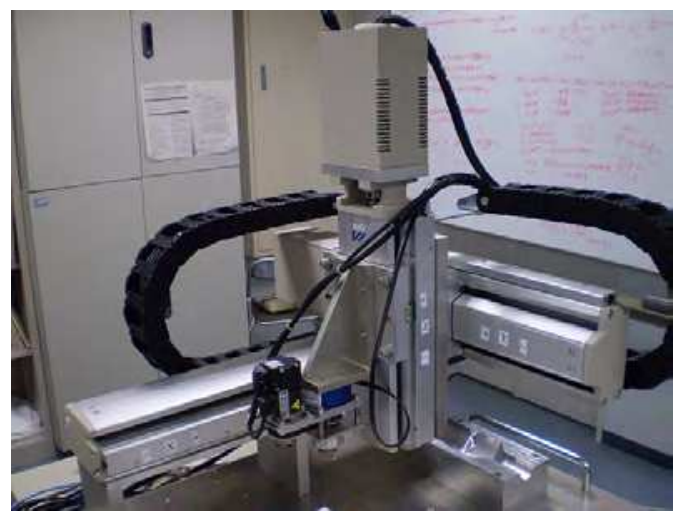

Fig. 2. Developed desktop Cartesian-type robot with abilities of compliant motion and stickslip motion. 


\section{Desktop Cartesian-Type Robot}

Figure 2 shows the developed desktop Cartesian-type robot consisting of three single-axis robots with position resolution of $1 \mu \mathrm{m}$. The size of the robot is $850 \times 645 \times 700 \mathrm{~mm}$. The single-axis robot is a position control device ISPA with high-precision resolution provided by IAI Corp., which is comprised of a base, linear guide, ball-screw, AC servo motor. The effective strokes in $\mathrm{x}-, \mathrm{y}$ - and $\mathrm{z}$-directions are 400, 300 and $100 \mathrm{~mm}$, respectively. The tool axis is designed to be parallel to the $\mathrm{z}$-axis of the robot. A thin wood stick tool is fixed to the tip through a compact force sensor with 3 degree-of-freedom. To regulate the rotation, a servo spindle is located parallel to the tool axis. The hardware block diagram of the robot is shown in Fig. 3. For example, if one pulse is given to $x-, y-$ and $z$-axis robots, then each single-axis robot can simultaneously move $1 \mu \mathrm{m}$, respectively. Figure 4 shows an example of the static relation between the position and contact force in case of using a wood stick tool, in which $\boldsymbol{\square}$ and show values in press and unpress motions, respectively.

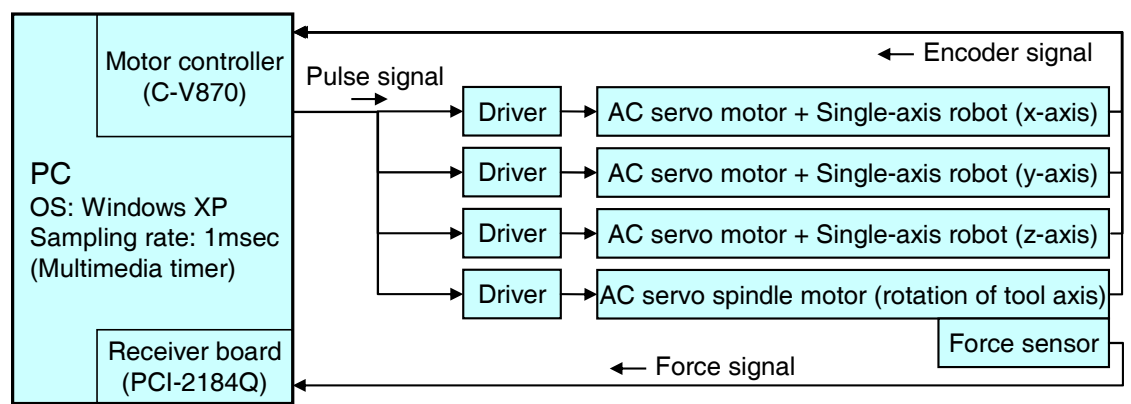

Fig. 3. Hardware block diagram of the proposed Cartesian-type robot composed of three single-axis robots with position resolution of $1 \mu \mathrm{m}$.

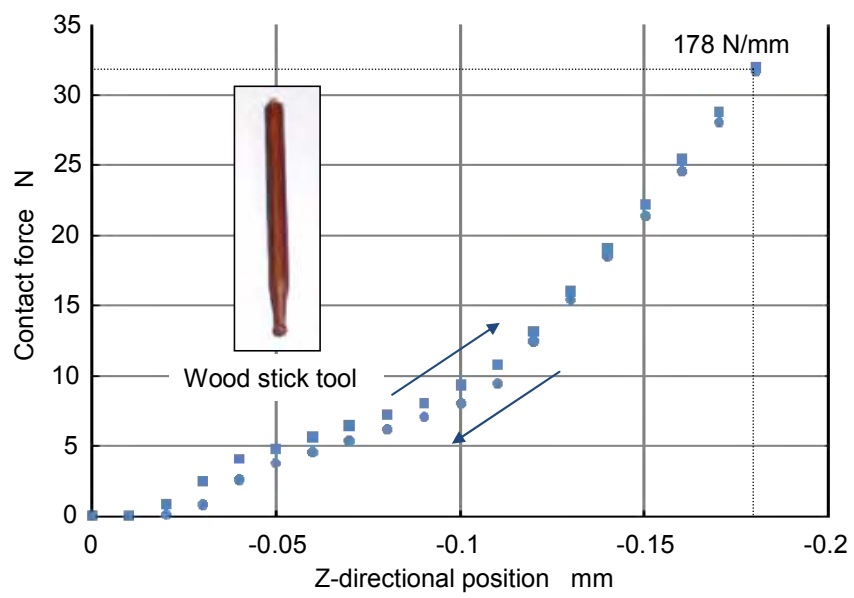

Fig. 4. An example of the static relation between position and contact force in case of using a wood stick tool, in which squares and circles show press and unpress motions, respectively. 
The quantity of the position is the z-directional component at the tip of the wood stick tool. The quantity of force is yielded by contacting the tool tip with a workpiece and is measured by the force sensor. The experiment was conducted while giving the relative position 0.01 $\mathrm{mm}$ in press motion and $-0.01 \mathrm{~mm}$ in unpress motion. In the experiment, the tool tip approached to an aluminum workpiece with a low speed, and after touching the workpiece, i.e., after detecting a small contact force, the tool tip was pressed to the workpiece with every $0.01 \mathrm{~mm}$. The graph drawn with $\mathbf{i n}$ Fig. 4 shows the relation the position and contact force. The force is about $32 \mathrm{~N}$ when the position of the tool tip is $-0.18 \mathrm{~mm}$, so that the effective stiffness within the range can be estimated about $178 \mathrm{~N} / \mathrm{mm}$. After the press motion, the tool tip was away from the workpiece once, and returned to the position again where $32 \mathrm{~N}$ had been obtained. After that, the tool tip was unpressed every $0.01 \mathrm{~mm}$. The graph drawn with $\bullet$ in Fig. 4 shows the relation of the position and contact force of this case. It is observed that the undesirable backlash is largely decreased compared with an articulated-type industrial robot (Nagata et al, 2008). It is expected that the force resolution about $0.178 \mathrm{~N}$ can be performed due to the position resolution of $1 \mu \mathrm{m}$.

\section{Compliant Motion of Wood Stick Abrasive Tool}

\subsection{Position/Force Control with Weak Coupling}

The basic finishing strategy is conducted along a continuous spiral path while performing stable polishing force control. In this section, the control system incorporated in the Cartesian-type robot is explained. The tool tip is controlled by the translational velocity ${ }^{W} \boldsymbol{v}(k)=\left[{ }^{W} v_{x}(k){ }^{W} v_{y}(k){ }^{W} v_{z}(k)\right]^{T}$, which is composed of three velocities as given by

$$
{ }^{W} \boldsymbol{v}(k)={ }^{W} \boldsymbol{v}_{t}(k)+{ }^{W} \boldsymbol{v}_{n}(k)+{ }^{W} \boldsymbol{v}_{p}(k)
$$

where $k$ denotes the discrete time; superscript ${ }^{W}$ denotes the work coordinate system. Note that the control system realizes $1 \mathrm{msec}$ sampling time by using the Windows multimedia timer. It is assumed that the polishing force is the resultant force of the contact force and kinetic friction forces. The kinetic friction forces are generated by Coulomb friction and viscous friction. The polishing force can be obtained as the resultant force of $x-, y-$ and $z-$ directional force sensor measurements. Figure 5 shows the proposed CAD/CAM-based position/force controller with weak coupling (Nagata et al, 2007). First of all, ${ }^{W} \boldsymbol{v}_{t}(k)$ is the manipulated variable generated from the feedforward control law based on cutter location data called the CL data. The CL data consist of sequential position and orientation components. ${ }^{W} \boldsymbol{v}_{t}(k)$ is the tangent velocity and written by

$$
{ }^{W} \boldsymbol{v}_{t}(k)=v_{\text {tangent }}(k) \frac{{ }^{W} t(k)}{\left\|{ }^{W} t(k)\right\|}
$$

where $v_{\text {tangent }}(k)$ is a velocity norm. ${ }^{W} t(k)$ is the tangent vector calculated by using the position compenents of two adjacent steps in the CL data. Also, ${ }^{W} \boldsymbol{v}_{n}(k)$ is the manipulated 
variable generated from the force feedback control law. ${ }^{W} \boldsymbol{v}_{n}(k)$ is the normal velocity and written by

$$
{ }^{W} \boldsymbol{v}_{n}(k)=v_{\text {normal }}(k){ }^{W} \boldsymbol{o}_{d}(k)
$$

where ${ }^{W} \boldsymbol{o}_{d}(k)$ is the normalized normal direction vector calculated by using the orientation compenents in CL data. The scalar $v_{\text {normal }}(k)$ representing the normal velocity is the output of the impedance model following force control law (Nagata et al, 2007) given by

$$
v_{\text {normal }}(k)=v_{\text {normal }}(k-1) e^{-\frac{B_{d}}{M_{d}} \Delta t}+\left(e^{-\frac{B_{d}}{M d} \Delta t}-1\right) \frac{K_{f}}{B_{d}} E_{f}(k)
$$

where $K_{f}$ is the force feedback gain, and impedance parameters $M_{d}$ and $B_{d}$ are the desired mass and damping coefficients, respectively. $\Delta t$ is the sampling time. Also, $E_{f}(k)$ is the error between the desired polishing force $F_{d}$ and the norm of force vector ${ }^{s} \boldsymbol{F}(k) \in \mathfrak{R}^{3}$ measure by the force sensor, which is given by

$$
E_{f}(k)=F_{d}-\|s \boldsymbol{F}(k)\|
$$

where superscript ${ }^{S}$ represents the sensor coordinate system. Further, ${ }^{W} \boldsymbol{v}_{p}(k)$ is the manipulated variable yielded by a position feedback control law based on a simple PI action, which is given by

$$
{ }^{W} \boldsymbol{v}_{p}(k)=\boldsymbol{S}_{p}\left(\boldsymbol{K}_{p} \boldsymbol{E}_{p}(k)+\boldsymbol{K}_{i} \sum_{n=1}^{k} \boldsymbol{E}_{p}(n)\right)
$$

where the switch matrix $\boldsymbol{S}_{p}=\operatorname{diag}\left(S_{p x}, S_{p y}, S_{p z}\right)$ makes the weak coupling control to the force control active or inactive in each direction; $\boldsymbol{E}_{p}(k)={ }^{W} \boldsymbol{x}_{d}(k)-{ }^{W} \boldsymbol{x}(k)$ is the position error. The desired position ${ }^{W} \boldsymbol{x}_{d}(k)$ is calculated by using the position components in CL data. ${ }^{W} \boldsymbol{x}(k)$ is the current position measured by the encoders of servo motor. $\boldsymbol{K}_{p}=\operatorname{diag}\left(K_{p x}, K_{p y}, K_{p z}\right)$ and $\boldsymbol{K}_{i}=\operatorname{diag}\left(K_{i x}, K_{i y}, K_{i z}\right)$ are proportional and integral gains for the position feedback control. Due to the weak coupling control, it is simultaneously realized that stable polishing force control and profiling control along a fine spiral path. 


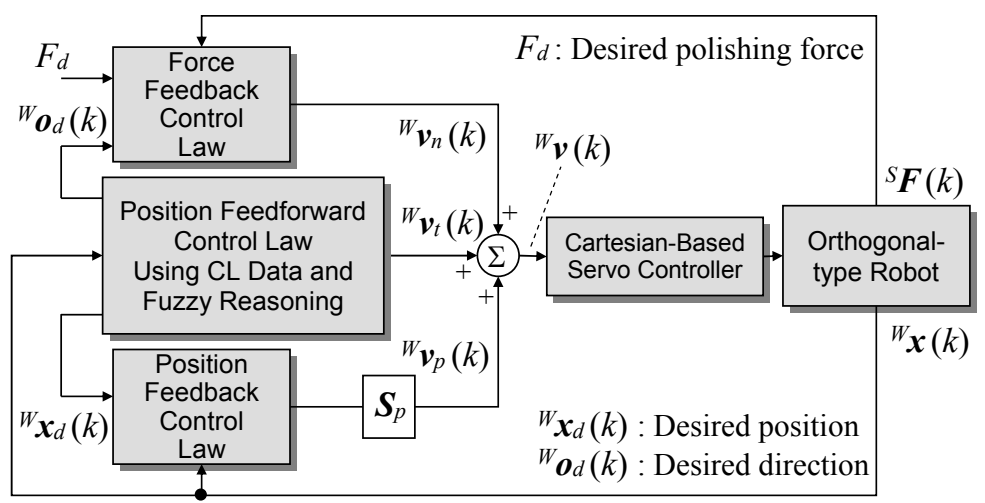

Fig. 5. CAD/CAM-based position/force controller with weak coupling.

\subsection{Tuning of Desired Damping}

Next, a tuning method of the desired damping is proposed by using the effective stiffness of the Cartesian-type robot. When the polishing force is controlled, the characteristics of force control system can be varied according to the combination of impedance parameters such as desired mass $M_{d}$ and damping $B_{d}$. In order to increase the force control stability the desired damping, which has much influence on force control stability, should be tuned suitably. In this section, a tuning method of the desired damping is proposed based on the critical damping condition with the effective stiffness of the Cartesian-type robot. Eq. (4) is derived from the following impedance model.

$$
M_{d}\left(\ddot{x}-\ddot{x}_{d}\right)+B_{d}\left(\dot{x}-\dot{x}_{d}\right)=K_{f}\left(F-F_{d}\right)
$$

where $\ddot{x}, \dot{x}$ and $F$ are the acceleration, velocity and force scalars in the direction of force control, respectively. $\ddot{x}_{d}, \dot{x}_{d}$ and $F_{d}$ are the desired acceleration, velocity and force scalars, respectively. When the force control is active, $\ddot{x}_{d}$ and $\dot{x}_{d}$ are set to zero. It is assumed that $F$ is the external force given by the environment and is modelled as

$$
F=-B_{m} \dot{x}-K_{m} x
$$

where $B_{m}$ and $K_{m}$ are the viscosity and stiffness coefficients of the environmnet, respectively. Eqs. (7) and (8) lead to the following second order lag system.

$$
\ddot{x}+\frac{B_{d}+K_{f} B_{m}}{M_{d}} \dot{x}+\frac{K_{f} K_{m}}{M_{d}} x=0
$$

The characteristics equation of Eq. (9) is written by 


$$
s^{2}+\frac{B_{d}+K_{f} B_{m}}{M_{d}} s+\frac{K_{f} K_{m}}{M_{d}}=0
$$

In this case, the damping coefficient $\zeta$ and natural frequency $\omega_{n}$ are given by

$$
\zeta=\frac{B_{d}+K_{f} B_{m}}{2 \sqrt{M_{d} K_{f} K_{m}}}, \quad \omega_{n}=\sqrt{\frac{K_{f} K_{m}}{M_{d}}}
$$

Further, solving Eq. (10) for $B_{d}$ using the critical damping condition, the following simple condition is obtained.

$$
B_{d}=2 \sqrt{M_{d} K_{f} K_{m}}-K_{f} B_{m}
$$

In profiling control experiment, the base value for the desired damping is calculated with Eq. (12). The desired damping should be fine-tuned around the base value according to the actual system.

\subsection{Finishing Experiment without Stick-Slip Motion Control}

In this subsection, the proposed Cartesian-type robot is applied to a finishing experiment of an LED lens mold. Figure 6 shows the model of an LED lens mold designed by the 3D CAD/CAM Pro/ENGINEER. The model is designed based on an edge of the mold profile. First of all, an inner edge is drawn in 2D. Then, z-axis is defined as shown in Fig. 6. The zaxis is called the spiral direction. After that, an inner surface can be created by revolving the edge around the $\mathrm{z}$-axis. Figure 7 shows the image of a spiral path generated from the mainprocessor of the CAM, which is used in the finishing experiment. The spiral path has sequential position and orientation components. The control parameters and finishing conditions tuned for the experiment are tabulated in Table 1.

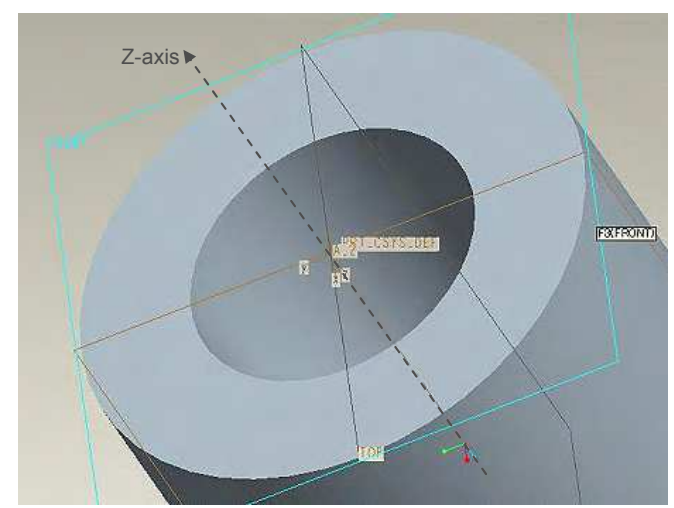

Fig. 6. 3D model of an LED lens mold designed by 3D CAD/CAM Pro/Engineer. 


\begin{tabular}{l|c}
\hline Conditions & Values \\
\hline Desired trajectory along curved surface & Spiral path \\
Pitch of spiral path & $0.01 \mathrm{~mm}$ \\
Radius of ball-end abrasive tool $R$ & $1 \mathrm{~mm}$ \\
Grain size of diamond lapping paste & $4 \mu \mathrm{m}$ \\
Desired polishing force $F_{d}$ & $5 \mathrm{~N}$ \\
Tool revolution per minute & $400 \mathrm{rpm}$ \\
Tangent directional velocity $\left\|{ }^{W} \boldsymbol{v}_{t}\right\|$ & $0.1 \mathrm{~mm} / \mathrm{s}$ \\
Desired mass coefficient $M_{d}$ & $0.01 \mathrm{~N} \cdot \mathrm{s}^{2} / \mathrm{mm}$ \\
Desired damping coefficient $B_{d}$ & $0.183 \mathrm{~N} \cdot \mathrm{s} / \mathrm{mm}$ \\
Force feedback gain $K_{f}$ & 0.01 \\
Diagonal elements of switch matrix $S_{p}$ & $0,0,1$ \\
Position feedback gain $K_{p x}, K_{p y}, K_{p z}$ & $0,0,0.001$ \\
Integral control gain $K_{i x}, K_{i y}, K_{i z}$ & $0,0,0.00001$ \\
Sampling time $\Delta t$ & $1 \mathrm{~ms}$ \\
\hline
\end{tabular}

Table 1. Control parameters and finishing conditions tuned for an experiment.

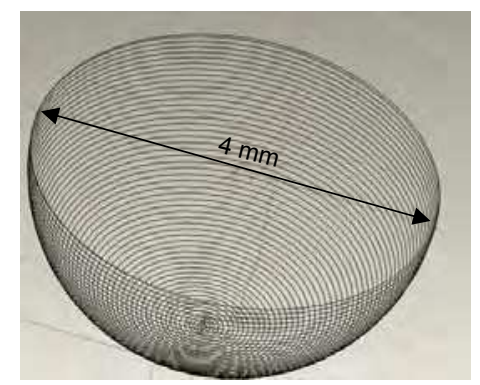

Fig. 7. Image of spiral path generated by using the main-processor of the CAM, which is used for the desired trajectory of the wood stick tool.

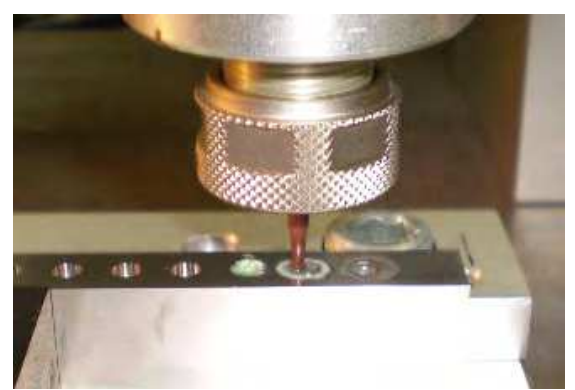

Fig. 8. Dexterous finishing scene by using the wood stick tool and diamond lapping paste.

Figure 8 shows the finishing scene of the LED lens mold, where a special oil including the diamond lapping paste is poured. The diameter of the concaved area is $4 \mathrm{~mm}$. In this case, a small ball-end tool lathed from a wood stick is used, whose tip diameter is $1 \mathrm{~mm}$. Actually, the tool tip moves along an inner path compared to the CL data used as a desired trajectory. The reason is that the position of the tool tip is corrected by the force feedback loop according to the diameter of the tool tip. 


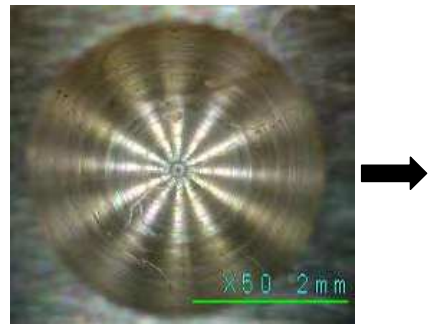

Before finishing

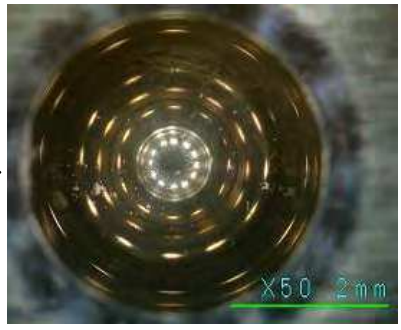

After finishing

Fig. 9. Finished surfaces before and after the finishing experiment.

Figure 9 shows the surfaces before and after the finishing process. It is observed that the concaved surface area has a good quality like a mirror surface reflecting the room lights. Although small oily spots are observed, they can be cleaned easily.

\section{Stick-Slip Motion of Wood Stick Abrasive Tool}

\subsection{Stick-Slip Motion Control}

In this section, the effectiveness of the tool's stick-slip motion is evaluated to improve the surface quality. Generally, the stick-slip motion is an undesirable phenomenon and should be eliminated in various precise machine tools. However, the proposed Cartesian-type robot employs a small stick-slip motion not only to partially improve the finishing quality but also to skillfully emphasize the polishing energy. Figure 10 shows the images of stick-slip motion seen like small vibrations along a straight path and a curved path. The stick-slip motion is given along curved surface and also to orthogonal directions to tool's tangent velocity ${ }^{W} \boldsymbol{v}_{t}(k)$. Here, how to generate small stick-slip motion vectors is explained in detail by using Fig. 11. In Fig. 11, point $\mathrm{O}$ is the origin in work coordinate system, where the tool tip initially contacts to the workpiece. Point $\mathrm{P}$ is the current contact point.

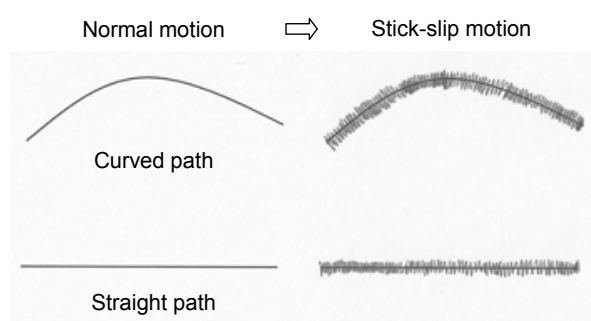

Fig. 10. Image of the small stick-slip motion seen like small vibrations for an abrasive tool. 


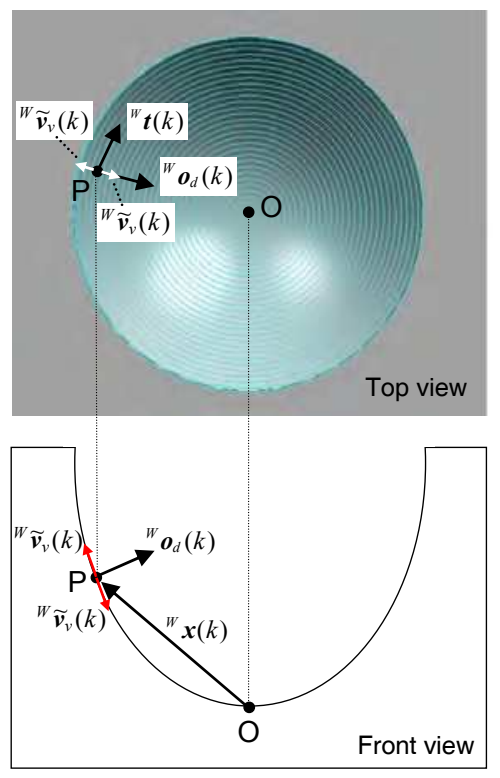

Fig. 11. Theoretical idea to calculate the stick-slip motion vector ${ }^{W} \boldsymbol{v}_{v}(k)$, where the directions of ${ }^{W} \boldsymbol{v}_{t}(k)$ and ${ }^{W} \boldsymbol{v}_{n}(k)$ are the same ones of ${ }^{W} \boldsymbol{t}(k)$ and ${ }^{W} \boldsymbol{o}_{d}(k)$, respectively ${ }^{W} \boldsymbol{x}(k)$ is the position vector given by ${ }^{W} \boldsymbol{x}(k)=\left[{ }^{W} x(k){ }^{W} y(k){ }^{W} z(k)\right]^{T}$ viewed from point $\mathrm{O} ;{ }^{W} \boldsymbol{o}_{d}(k)$ is the normalized normal vector at the point $\mathrm{P}$ written by ${ }^{W} \boldsymbol{o}_{d}(k)=\left[{ }^{W} \boldsymbol{O}_{d x}(k){ }^{W} \boldsymbol{O}_{d y}(k){ }^{W} \boldsymbol{O}_{d z}(k)\right]^{T}$ and is calculated with the orientation components in CL data; ${ }^{W} \boldsymbol{t}(k)=\left[{ }^{W} t_{x}(k){ }^{W} t_{y}(k){ }^{W} t_{z}(k)\right]^{T}$ is the tangent vector at the point $\mathrm{P}$. Here, it is assumed that ${ }^{W} \boldsymbol{v}_{v}(k)=\left[{ }^{W} v_{v x}(k){ }^{W} v_{v y}(k){ }^{W} v_{v z}(k)\right]^{T}$ is a small stick-slip vector to be considered in this section.

In this example, the tool approaches to the workpiece with a low speed and follow the spiral path after contacting the point O. Because ${ }^{W} \boldsymbol{v}_{v}(k)$ is perpendicular to ${ }^{W} \boldsymbol{o}_{d}(k)$, the following relation is obtained.

$$
{ }^{W} v_{v x}(k){ }^{W} o_{d x}(k)+{ }^{W} v_{v y}(k){ }^{W} o_{d y}(k)+{ }^{W} v_{v z}(k){ }^{W} o_{d z}(k)=0
$$

Also, ${ }^{W} \boldsymbol{v}_{v}(k)$ and ${ }^{W} \boldsymbol{t}(k)$ are orthogonal each other, so that

$$
{ }^{W} v_{v x}(k){ }^{W} t_{x}(k)+{ }^{W} v_{v y}(k){ }^{W} t_{y}(k)+{ }^{W} v_{v z}(k){ }^{W} t_{z}(k)=0
$$

Further, ${ }^{W} \boldsymbol{v}_{v}(k)$ is located in a plane which includes both ${ }^{W} \boldsymbol{o}_{d}(k)$ and ${ }^{W} \boldsymbol{x}(k)$, so that the components of ${ }^{W} \boldsymbol{v}_{v}(k)$ are represented by 


$$
\begin{aligned}
& { }^{W} v_{v x}(k)=i^{W} o_{d x}(k)+j^{W} x(k) \\
& { }^{W} v_{v y}(k)=i^{W} o_{d y}(k)+j^{W} y(k) \\
& { }^{W} v_{v z}(k)=i^{W} o_{d z}(k)+j^{W} z(k)
\end{aligned}
$$

where $i$ and $j$ are real numbers. By solving Eqs. (13), (14), (15), (16) and (17), ${ }^{W} v_{v x}(k)$, ${ }^{W} v_{v y}(k)$ and ${ }^{W} v_{v z}(k)$ can be obtained. Here, however, a simpler calculation is used. First of all, substituting Eqs. (15), (16) and (17) into Eq. (13) leads to

$$
\begin{aligned}
i\left(\left({ }^{W} o_{d x}(k)\right)^{2}+\left({ }^{W} o_{d y}(k)\right)^{2}+\left({ }^{W} o_{d z}(k)\right)^{2}\right) \\
\quad+j\left({ }^{W} o_{d x}(k){ }^{W} x(k)+{ }^{W} o_{d y}(k){ }^{W} y(k)+{ }^{W} o_{d z}(k){ }^{W} z(k)\right)=0
\end{aligned}
$$

$\left\|{ }^{W} \boldsymbol{o}_{d}(k)\right\|=1$ further leads to

$$
i=-j\left({ }^{W} o_{d x}(k){ }^{W} x(k)+{ }^{W} o_{d y}(k){ }^{W} y(k)+{ }^{W} o_{d z}(k){ }^{W} z(k)\right)
$$

Accordingly, by giving Eq. (19) into Eqs. (15), (16) and (17), the following equations are obtained.

$$
\begin{aligned}
& { }^{W} v_{v x}(k)=j\left({ }^{W} x(k)-\left[{ }^{W} o_{d x}(k){ }^{W} x(k)+{ }^{W} o_{d y}(k){ }^{W} y(k)+{ }^{W} o_{d z}(k){ }^{W} z(k)\right]{ }^{W} o_{d x}(k)\right) \\
& { }^{W} v_{v y}(k)=j\left({ }^{W} y(k)-\left[{ }^{W} o_{d x}(k){ }^{W} x(k)+{ }^{W} o_{d y}(k){ }^{W} y(k)+{ }^{W} o_{d z}(k){ }^{W} z(k)\right]{ }^{W} o_{d y}(k)\right)
\end{aligned}
$$

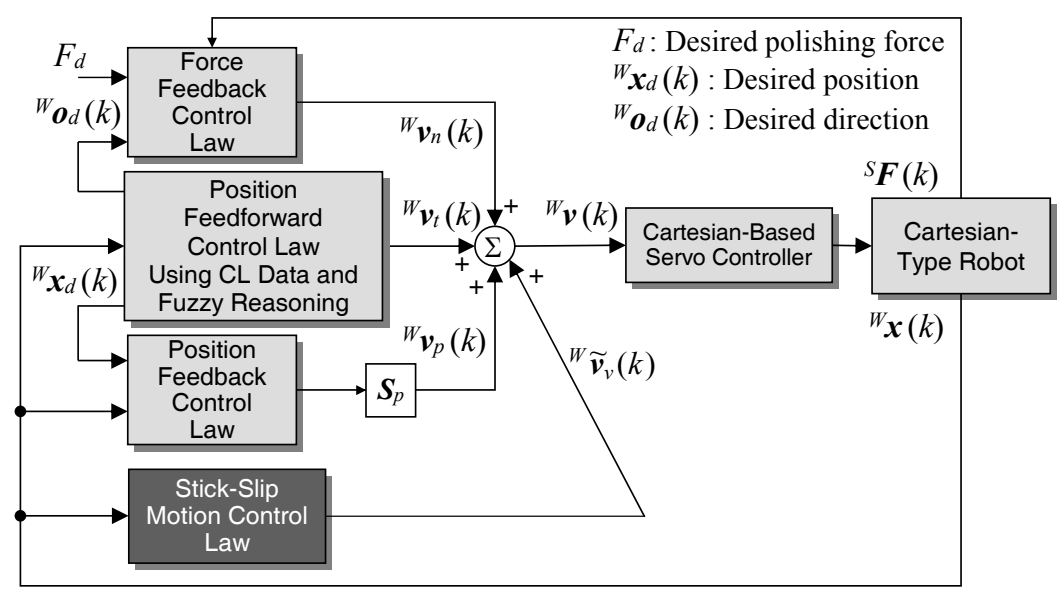

Fig. 12. Proposed CAD/CAM-based position/force controller with stick-slip motion. 


$$
{ }^{W} v_{v z}(k)=j\left({ }^{W} z(k)-\left[{ }^{W} o_{d x}(k){ }^{W} x(k)+{ }^{W} o_{d y}(k){ }^{W} y(k)+{ }^{W} o_{d z}(k){ }^{W} z(k)\right]{ }^{W} o_{d z}(k)\right)
$$

Because both ${ }^{W} \boldsymbol{o}_{d}(k)$ and ${ }^{W} \boldsymbol{x}(k)$ are known, ${ }^{W} \boldsymbol{v}_{v}(k)$ can be normalized as ${ }^{W} \boldsymbol{v}_{v}(k) /\left\|{ }^{W} \boldsymbol{v}_{v}(k)\right\|$. Further, by using a scalar $K_{v}$ and a $\operatorname{sign} \operatorname{SIGN}(k)$, the stick-slip motion vector is finally obtained as

$$
{ }^{W} \widetilde{\boldsymbol{v}}_{v}(k)=\operatorname{SIGN}(k) K_{v} \frac{{ }^{W} \boldsymbol{v}_{v}(k)}{\left\|{ }^{W} \boldsymbol{v}_{v}(k)\right\|}
$$

where $\operatorname{SIGN}(k)$ is given by

$$
\operatorname{SIGN}(k)=\left\{\begin{aligned}
1 & \text { if } k=\text { odd number } \\
-1 & \text { otherwise }
\end{aligned}\right.
$$

${ }^{W} \widetilde{\boldsymbol{v}}_{v}(k)$ is a velocity vector to yield another polishing energy, and which is given to the tool tip alternately changing the direction every sampling period. Figure 12 shows the block diagram of the controller with the stick-slip motion control method. As can be seen from Eqs. (2) and (3), the directions of ${ }^{W} \boldsymbol{v}_{t}(k)$ and ${ }^{W} \boldsymbol{v}_{n}(k)$ are the same ones of ${ }^{W} \boldsymbol{t}(k)$ and ${ }^{W} \boldsymbol{o}_{d}(k)$, respectively. Also, ${ }^{W} \boldsymbol{v}_{p}(k)$ is generated in the direction of z-axis called the spiral direction as shown in Fig. 6.

\subsection{Experiment of Stick-Slip Motion Control}

Next, the effectiveness of the stick-slip motion control is examined through an actual finishing test. In the conventional finishing method shown in Fig. 5, the proposed stick-slip motion was not applied. Although the concaved area after finishing shown in Fig. 9 may be seen as a high quality surface, uneven lines are observed as shown in Fig. 13, in which small cusps still remain.

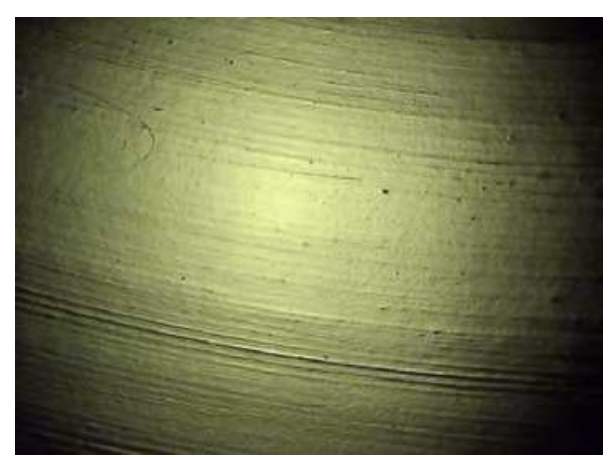

Fig. 13. Large scale photo of the LED lens mold, where undesirable small cusps still remain on the surface. 


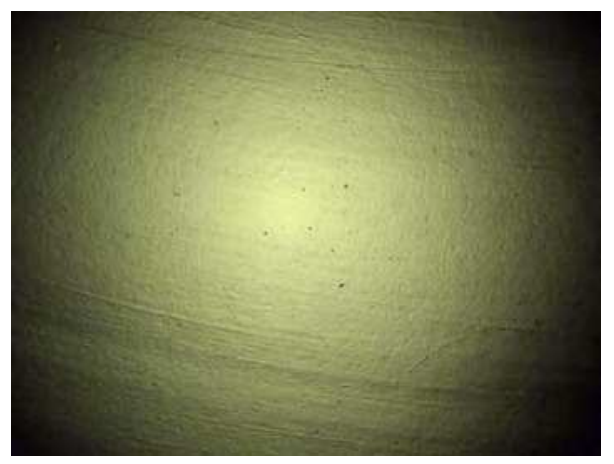

Fig. 14. Large scale photo of the LED lens mold after the finishing process by partially using the proposed stick-slip motion control.

Figure 14 shows the large scale photo of the LED lens mold after the finishing process by partially using the proposed stick-slip motion control. It is observed that the undesirable cusps can be removed uniformly. It has been confirmed from the result that the proposed finishing strategy by using the stick-slip motion control has a promising effectiveness to achieve a higher quality surface.

\section{Conclusions and Future Works}

The final goal of this study is the development of a novel Cartesian-type robot with compliance controllability that can be applied to from the cusp mark removing process to the finishing process for mirror-like surface of LED lens molds. In this chapter, a desktop Cartesian-type robot with 3 degree-of-freedom was first designed by combining three single-axis robots with a high position resolution of $1 \mu \mathrm{m}$. The position resolution and force resolution, and effective stiffness were examined through a simple contact experiment, so that the force resolution of $0.178 \mathrm{~N}$ was obtained due to the position resolution of $1 \mu \mathrm{m}$. Next, a hybrid position/force controller with compliance controllability was proposed for the finishing task of LED lens molds, in which position control, force control or their weak coupling control can be selected according to each finishing strategy. A systematic tuning method of the desired damping was also considered. The desired damping was calculated from the critically damped condition using the static relation between the position and force. Further, a stick-slip motion control for a wood stick tool was developed to finely improve the finishing quality. The proposed desktop Cartesian-type robot using these peripheral techniques was applied to a finishing experiment of an LED lens mold, so that the high performance and promise were successfully confirmed. In future work, we plan to consider other potential applications using the Cartesian-type robot.

\section{References}

Oba, T.; Kawai, T.; Takino, H. \& Takeuchi, Y. (2008). Ultraprecision multi-axis control machining of spherical micro-lens array mold. Proceedings of JSPE Semestrial Meeting, B67, Tohoku University, September 2008. 
Kuriyagawa, T.; Saeki, M. \& Syoji, K. (2002). Electrorheological fluid-assisted ultra-precision polishing for small three-dimensional parts. Precision engineering, Vol. 26, No. 4, pp. 370-380.

Suzuki, H.; Moriwaki, T.; Okino, T. \& Ando Y. (2006). Development of ultrasonic vibration assisted polishing machine for micro aspheric die and mold. CIRP Annals Manufacturing Technology, Vol. 55, No. 1, pp. 385-388.

Bilkay, O. \& Anlagan, O. (2004). Computer simulation of stick-slip motion in machine tool slideways. Tribology International, Vol. 37, No. 4, pp. 347-351.

Mei, X.; Tsutsumi, M.; Tao, T. \& Sun, N. (2004). Study on the compensation of error by stickslip for high-precision table. International Journal of Machine tools \& Manufacture, Vol. 44, No. 5, pp. 503-510.

Nagata, F.; Hase, T.; Haga, Z.; Omoto, M. \& Watanabe, K. (2007). CAD/CAM-based position/force controller for a mold polishing robot. Mechatronics, Vol. 17, No. 4-5, pp. 207-216.

Nagata, F.; Hase, T.; Haga, Z.; Omoto, M. \& Watanabe, K. (2008). CAD/CAM-based Position/Force Control for a Ball-end Abrasive Tool and Its Application to an Industrial Robot. Journal of Advanced Mechanical Design, Systems, and Manufacturing, Vol. 2, No. 4, pp. 742-752. 


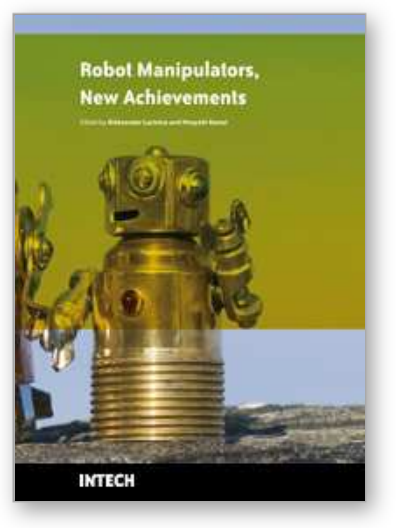

\author{
Robot Manipulators New Achievements \\ Edited by Aleksandar Lazinica and Hiroyuki Kawai
}

ISBN 978-953-307-090-2

Hard cover, 718 pages

Publisher InTech

Published online 01, April, 2010

Published in print edition April, 2010

Robot manipulators are developing more in the direction of industrial robots than of human workers. Recently, the applications of robot manipulators are spreading their focus, for example Da Vinci as a medical robot, ASIMO as a humanoid robot and so on. There are many research topics within the field of robot manipulators, e.g. motion planning, cooperation with a human, and fusion with external sensors like vision, haptic and force, etc. Moreover, these include both technical problems in the industry and theoretical problems in the academic fields. This book is a collection of papers presenting the latest research issues from around the world.

\title{
How to reference
}

In order to correctly reference this scholarly work, feel free to copy and paste the following:

Fusaomi Nagata, Shintaro Tani and Takanori Mizobuchi, Tetsuo Hase, Zenku Haga and Keigo Watanabe (2010). Desktop Cartesian-Type Robot with Abilities of Compliant Motion and Stick-Slip Motion, Robot Manipulators New Achievements, Aleksandar Lazinica and Hiroyuki Kawai (Ed.), ISBN: 978-953-307-090-2, InTech, Available from: http://www.intechopen.com/books/robot-manipulators-new-achievements/desktopcartesian-type-robot-with-abilities-of-compliant-motion-and-stick-slip-motion

\section{INTECH}

open science | open minds

\section{InTech Europe}

University Campus STeP Ri

Slavka Krautzeka 83/A

51000 Rijeka, Croatia

Phone: +385 (51) 770447

Fax: +385 (51) 686166

www.intechopen.com

\section{InTech China}

Unit 405, Office Block, Hotel Equatorial Shanghai

No.65, Yan An Road (West), Shanghai, 200040, China

中国上海市延安西路65号上海国际贵都大饭店办公楼405单元

Phone: +86-21-62489820

Fax: +86-21-62489821 
(C) 2010 The Author(s). Licensee IntechOpen. This chapter is distributed under the terms of the Creative Commons Attribution-NonCommercialShareAlike-3.0 License, which permits use, distribution and reproduction for non-commercial purposes, provided the original is properly cited and derivative works building on this content are distributed under the same license. 